\title{
Screening of Maize Inbred Lines Under Artificial Epiphytotic Condition for their Reaction to Turcicum Leaf Blight (TLB) and Common Leaf Rust (CLR)
}

\author{
Alemayehu Hailu*, Tajudin Aliyi and Bayoush Birke \\ Ethiopian Institute of Agricultural Research, Ambo Plant Protection Research Center, Ethiopia
}

Submission: December 16, 2017, Published: December 11, 2018

"Corresponding author: Alemayehu Hailu, Ethiopian Institute of Agricultural Research, Ambo Plant Protection Research Center, P.0. Box 37, Ambo, Ethiopia

\begin{abstract}
Common leaf rust (Puccinia sorghi Schw) and Turcicum leaf blight (Exserohilum trurcicum) is the major foliar fungal diseases of maize in Ethiopia causing yield losses in the range of $12 \%$ to $61 \%$ rely up on the genotypes. Screening was done on 178 (106 QPM and 72 non-QPM or normal maize lines) maize inbred lines against CLR and TLB diseases in order to know the reaction of those maize lines for two consecutive years, at Ambo plant protection research center (TLB and CLR) and Bako agricultural research center (TLB only), on station experimental fields. A randomized complete block design was used. Among 73(33 QPM and 20 non- QPM) maize lines, resistant and susceptible responses were recorded on 42(33QPM and 9 non- QPM) and 3 (1 QPM and 2 non- QPM) lines for TLB disease, respectively. Out of 105 (53 QPM and 52 nonQPM) maize lines, resistant and susceptible responses were recorded on 33 (11 QPM and 22 non-QPM) and 4 (QPM only) lines for CLR disease, in that order. Therefore, attention should be given for both diseases during screening in order to develop resistant maize varieties for both maize types. Those selected resistance maize lines from this screening should be used in breeding program and finding of resistant maize lines for both diseases will be continued using conventional and molecular methods.
\end{abstract}

Keywords: Common leaf rust; Puccinia sorghi Schw; Turcicum leaf blight; Exserohilum trurcicum;

Resistant response; Susceptible response; Maize lines

Abbreviations: TLB: Turcicum Leaf Blight; CLR: Common Leaf Rust; RCBD: Randomized Complete Block Design; MR: Moderately Resistant; AUDPC: Area Under Disease Progress Curve; MS: Moderately Susceptible

\section{Introduction}

Maize (Zea mays L.) is an important staple food crop and provides raw materials for the livestock and many agro-allied industries in the world [1]. It is a staple food for several million people in the developing world where they derive their protein and calorie requirements from it.

Maize is among the leading cereal crops selected to achieve food self-sufficiency in Ethiopia [2]. Although, improved cultivars have been largely included in the national extension package, the national average yield of maize is only 3.45 tons/ha [3], which is far below the world average of 5.5 tons/ha. The low yield is attributed to a number of factors such as Biotic (Diseases, insect pests, and weeds), abiotic (moisture, soil fertility, etc). Among biotic factors, foliar diseases such as turcicum leaf blight (Exserohilum trurcicum) and common rust (Puccinia sorghi Schw) are generally among the important constraints in tropical maize production [4]. In Ethiopia, the two diseases can cause yield loss in the range of 12 to 61.0 percent depending up on the genotype (Unpublished data). Previously the disease was limited to specific areas and varieties, but currently the disease become very important almost in all maize growing agro-ecologies due to climate change and pathogens virulent and/or avirulent shifts.

CLR and TLB can be effectively controlled by growing resistant varieties. More farmers need to adopt resistant maize varieties in order to withstand future CLR and TLB outbreaks in Ethiopia. Genetic resistance is the safest and best control strategy for resource-poor farmers in addition to being profitable option for farmers that can multiply seed [5]. Thus, the objective of this study was to evaluate the reaction of maize lines against CLR and TLB under field conditions with artificial inoculation.

\section{Materials and Methods}

\section{Maize field evaluation}

The experiment was conducted for two consecutive years (2015 and 2016 growing seasons) at Ambo (TLB and CLR) and 
Bako (TLB only). Maize lines were evaluated against CLR and TLB diseases. The treatment was arranged following a randomized complete block design (RCBD) with three replications for two disease types, separately. The plots were ploughed with tractor and disc harrowed twice before planting. The distance between rows and plants were $75 \mathrm{~cm}$ and $25 \mathrm{~cm}$, respectively. All plots were planted by hand with two seeds per hole. Inorganic fertilizer (Dap \& Urea) and all agronomic practices were applied based on the area recommendations.

\section{Inoculation and disease assessment}

Inoculation ways: The TLB pathogens were isolated by collecting diseased maize leaf lesions and placing in a moist chamber. After two-three days newly formed spores on the surface of the lesions was picked up with the help of fine flattened needle under a dissecting microscope placed in a droplet of sterile water and streak across the surface hardened, acidified water agar in petri-plates. After $6 \mathrm{hrs}$ the spores start to germinate, and it was cut out of the agar and transferred to hard, acidified PDA. After two weeks of incubation at $20-25{ }^{\circ} \mathrm{C}$, this culture was transferred to fresh plates of acidified PDA for multiplication. When the fungus growth was covered the surface of petri-plate fully, the cultures were ready for use. The spore (TLB) suspension@ 60,000spores/ $\mathrm{ml}$ was applied in the whorl using atomizer hand sprayers. Inoculation was made twice a week for three weeks, when plants were $30-45 \mathrm{~cm}$ high.

Inoculum (rust) was collected naturally infected leaves showing large number of pustules. Collection of rust uredospore was done by lightly tapping the leaves in to a cup or a suitable container. The spores were dried and kept in tightly sealed glass jars and stored at minus $20^{\circ} \mathrm{C}$. Maize plants under field conditions were inoculated first time at around 6-8 leaf stage and it repeated within 2 weeks. Rust spore suspension @ 60,000 spores/ml was prepared and applied in the whorl using hand atomizer. To avoid spores clump together on the upper surface of the water, the spore suspension was agitated (stirred) continuously and tween 20 was added in the solutions.

\section{Disease assessment}

\section{Disease severity estimation}

Maize lines were pheno typed for TLB and rust severity when the diseases are appeared using standard 1-5 scale, 1 being complete resistant and 5 being the complete susceptible [6]. Based on this rating scale over two years, maize lines were categorized into four groups namely, resistant (R) genotypes with a score < 2.0; moderately resistant (MR) 2.1-3.0; moderately susceptible (MS) 3.1-3.5 and highly susceptible (S) > 3.5.

\section{Area under disease progress curve (AUDPC)}

AUDPC (\% day) was calculated from severity and it was recorded 6 times at ten-day interval starting from one set of disease for each year. Disease severity was recorded from 10 randomly selected and tagged plants in each plot for AUDPC calculation. AUDPC was calculated using the formula suggested by Wilcoxson et al. [7].

$$
A U D P C=\sum_{i=1}^{n-1} 0.5\left(X_{i+1}+X_{i}\right)\left(t_{i+1}-t_{i}\right)
$$

Where, $\mathrm{X}_{\mathrm{i}}$ is the cumulative disease severity expressed as a proportion at the $\mathrm{i}^{\text {th }}$ observation; $\mathrm{t}_{\mathrm{i}}$ is the time (days after planting) at the $\mathrm{i}^{\text {th }}$ observation and $\mathrm{n}$ is total number of observations.

\section{Data analysis}

Analysis of variance (ANOVA) was used for disease data as randomized block design (RCBD) and following the procedure described by Gomez \& Gomez [8], using SAS computer software. Mean separation was done based on LSD at 5\% probability level. Disease data was analyzed after checking for good fitness to ANOVA.

\section{Result and Discussion}

Table 1: Reaction of Maize (QPM) lines for TLB disease at Ambo and Bako, in 2015.

\begin{tabular}{|c|c|c|c|c|c|}
\hline \multirow{2}{*}{ S.no } & \multirow{2}{*}{ Maize Lines } & \multicolumn{2}{|c|}{ Severity (1-5 scales) } & \multicolumn{2}{|c|}{ Maize Line Responses } \\
\hline & & Ambo & Bako & Ambo & Bako \\
\hline 1 & $\begin{array}{l}\text { [KIT/SNSYN[N3/TUX]]c1F1-\#\#(GLS=1.5)-31-17-1-1 /CM- } \\
\text { L144(BC2)-31-14-1-3-2-2-\#-1-1 }\end{array}$ & 2.3 & 2.04 & MR & MR \\
\hline 2 & $\begin{array}{l}\text { [KIT/SNSYN[N3/TUX]]c1F1-\#\#(GLS=2)-29-35-2-3/CM- } \\
\text { L144(BC2)-29-24-1-2-1-3-\#-3-1 }\end{array}$ & 2.3 & 2.5 & MR & MR \\
\hline 3 & $\begin{array}{l}\text { [KIT/SNSYN[N3/TUX]]c1F1-\#\#(GLS=2)-29-35-2-3/CM- } \\
\text { L144(BC2)-29-24-1-3-1-1-\#-2-1 }\end{array}$ & 1.5 & 1.9 & $\mathrm{R}$ & $\mathrm{R}$ \\
\hline 4 & $\begin{array}{l}\text { [KIT/SNSYN[N3/TUX]]c1F1-\#\#(GLS=2)-29-35-2-3/CM- } \\
\text { L144(BC2)-29-24-1-3-2-2-\#-1-1 }\end{array}$ & 2.2 & 2.04 & MR & MR \\
\hline 5 & $\begin{array}{l}\text { [KIT/SNSYN[N3/TUX]]c1F1-\#\#(GLS=2)-29-35-2-3/CM- } \\
\text { L144(BC2)-29-24-1-3-3-1-\#-1-1 }\end{array}$ & 1.9 & 2.8 & $\mathrm{R}$ & MR \\
\hline 6 & $\begin{array}{l}\text { [KIT/SNSYN[N3/TUX]]c1F1-\#\#(GLS=2)-29-35-2-3/CM- } \\
\text { L144(BC2)-29-24-1-3-3-3-\#-2-1 }\end{array}$ & 1.7 & 1.9 & $\mathrm{R}$ & $\mathrm{R}$ \\
\hline 7 & $\begin{array}{l}\text { [POOL9Ac7-SR(BC2)]FS211-1SR-1-1-1-\#/ } \\
\text { CML144(BC2)-14-21-1-3-2-2-\#-1-1 }\end{array}$ & 1.8 & 1.9 & $\mathrm{R}$ & $\mathrm{R}$ \\
\hline 8 & $\begin{array}{l}\text { [POOL9Ac7-SR(BC2)]FS211-1SR-1-1-1-\#/ } \\
\text { CML144(BC2)-14-21-2-1-4-1-\#-2-1 }\end{array}$ & 1.7 & 1.8 & $\mathrm{R}$ & $\mathrm{R}$ \\
\hline
\end{tabular}




\section{Agricultural Research \& Technology: Open Access Journal}

\begin{tabular}{|c|c|c|c|c|c|}
\hline 9 & $\begin{array}{l}\text { [POOL9Ac7-SR(BC2)]FS211-1SR-1-1-1-\#/ } \\
\text { CML144(BC2)-14-21-2-4-5-2-\#-4-1 }\end{array}$ & 1.8 & 2.5 & $\mathrm{R}$ & MR \\
\hline 10 & [POOL9Ac7-SR(BC2)]FS211-1SR-1-1-1-\#/CML144(BC2)-14-8-4-2-1-3-\#-1-1 & 1.9 & 2.5 & $\mathrm{R}$ & MR \\
\hline 11 & [POOL9Ac7-SR(BC2)]FS211-1SR-1-1-1-\#/CML144(BC2)-14-8-4-2-2-1-\#-2-1 & 1.9 & 2.4 & $\mathrm{R}$ & MR \\
\hline 12 & [POOL9Ac7-SR(BC2)]FS211-1SR-1-1-1-\#/CML144(BC2)-14-8-4-2-2-4-\#-3-1 & 2.4 & 2.2 & MR & MR \\
\hline 13 & [POOL9Ac7-SR(BC2)]FS211-1SR-1-1-1-\#/CML144(BC2)-14-8-4-2-3-3-\#-1-1 & 1.9 & 2.5 & $\mathrm{R}$ & MR \\
\hline 14 & [POOL9AC7-SR(BC2)]FS211-1SR-1-1-1-\#/CML144(BC2)-14-8-4-2-4-1-\#-1-1 & 1.5 & 2.2 & $\mathrm{R}$ & MR \\
\hline 15 & [POOL9Ac7-SR(BC2)]FS211-1SR-1-1-1-\#/CML144(BC2)-14-8-4-3-2-4-\#-1-1 & 1.5 & 1.7 & $\mathrm{R}$ & $\mathrm{R}$ \\
\hline 16 & [POOL9Ac7-SR(BC2)]FS211-1SR-1-1-1-\#/CML144(BC2)-14-8-4-3-3-4-\#-2-1 & 1.4 & 1.5 & $\mathrm{R}$ & $\mathrm{R}$ \\
\hline 17 & [POOL9Ac7-SR(BC2)]FS211-1SR-1-1-1-\#/CML144(BC2)-14-8-4-3-4-2-\#-3-1 & 1.5 & 1.7 & $\mathrm{R}$ & $\mathrm{R}$ \\
\hline 18 & [POOL9Ac7-SR(BC2)] FS48-1-1-1-1-1-\#/CML144(BC2)-6-25-2-2-4-1-\#-1-1 & 1.5 & 1.7 & $\mathrm{R}$ & $\mathrm{R}$ \\
\hline 19 & [POOL9Ac7-SR(BC2)] FS48-1-1-1-1-1-\#/CML144(BC2)-6-25-2-2-4-1-\#-5-1 & 1.6 & 1.9 & $\mathrm{R}$ & $\mathrm{R}$ \\
\hline 20 & [POOL9Ac7-SR(BC2)] FS48-1-1-3-1-\#/CML144(BC2)-15-8-1-1-3-2-\#-1-1 & 1.4 & 1.7 & $\mathrm{R}$ & $\mathrm{R}$ \\
\hline 21 & [POOL9Ac7-SR(BC2)] FS59-2-2-1-1-\#/CML144(BC2)-9-5-2-1-3-1-\#-4 & 1.7 & 1.9 & $\mathrm{R}$ & $\mathrm{R}$ \\
\hline 22 & $\begin{array}{c}\text { [KIT/SNSYN[N3/TUX]]c1F1-\#\#(GLS=2.5)-32-1-1-\#/CML176BC1F1-12-1-3- } \\
\text { 2-1-\#-1-B }\end{array}$ & 1.6 & 1.7 & $\mathrm{R}$ & $\mathrm{R}$ \\
\hline 23 & $\begin{array}{c}{[\mathrm{KIT} / \mathrm{SNSYN}[\mathrm{N} 3 / \mathrm{TUX}]] \mathrm{c} 1 \mathrm{~F} 1-\# \#(\mathrm{GLS}=2.5)-32-1-1-\# / \mathrm{CML176BC1F1-12-1-3-}} \\
4-2-\#-1-\mathrm{B}\end{array}$ & 1.5 & 2.1 & $\mathrm{R}$ & MR \\
\hline 24 & [POOL9Ac7-SR(BC2)] FS60-2-1-1-1-\#/CML176(BC2)-1-3-2-3-3-\#-2-B & 2.2 & 3.6 & MR & S \\
\hline 25 & [POOL9Ac7-SR(BC2)] FS60-2-1-1-1-\#/CML176(BC2)-1-3-2-3-4-\#-1-B & 1.8 & 2.6 & $\mathrm{R}$ & MR \\
\hline 26 & [POOL9Ac7-SR(BC2)] FS60-2-1-1-1-\#/CML176(BC2)-1-3-2-3-5-\#-1-B & 1.7 & 2.5 & $\mathrm{R}$ & MR \\
\hline 27 & [POOL9Ac7-SR(BC2)] FS60-2-3-1-3-1-\#/CML176(BC2)-3-1-3-3-3-\#-1-B & 1.8 & 1.5 & $\mathrm{R}$ & $\mathrm{R}$ \\
\hline 28 & SADVLA/CML154 BC2F54-4-1-B-\#-4-\# & 2.8 & 2.8 & MR & MR \\
\hline 29 & SADVLA/CML154 BC2F41-1-12-B-\#-\#-\# & 1.4 & 2.4 & $\mathrm{R}$ & MR \\
\hline 30 & SADVLA/CML154 BC2F37-2-1-B-\#-\#-\# & 1.6 & 1.7 & $\mathrm{R}$ & $\mathrm{R}$ \\
\hline 31 & P502 SR/CML384X176.....98-2-1-2 BC2F4-1-3-B-\#-\#-\# & 1.6 & 2.3 & $\mathrm{R}$ & MR \\
\hline 32 & CML176 & 2.5 & 2.4 & MR & MR \\
\hline 33 & CML491 & 1.6 & 2.5 & $\mathrm{R}$ & MR \\
\hline
\end{tabular}

Among 33 tested QPM maize lines, totally 26 (78.8\%) lines were showed resistant responses for Turcicum leaf blight at Ambo and Bako in 2015 (Table 1). Among26 QPM resistance maize lines, 14 (53.8\%) resistance maize lines were obtained both from Bako and Ambo in the same screening season, whereas 12 (46.2) resistance maize lines were got only from Ambo screening site., in 2015 (Table 1). Moderately, responses were recorded at Ambo and Bako on seven and eighteen maize lines in 2015, respectively. Only one QPM maize line showed susceptible response at Bako experimental site (Table 1).

Table 2: Reaction of Maize (QPM) lines for Common leaf rust disease at Ambo, in 2015.

\begin{tabular}{|c|c|c|c|}
\hline S.no & Maize lines & Severity (1-5 Scales) & Line Response \\
\hline 1 & $\begin{array}{c}\text { [KIT/SNSYN[N3/TUX]]c1F1-\#\#(GLS=1.5)-31-17-1-1 /CM- } \\
\text { L144(BC2)-31-14-1-3-2-2-\#-1-1 }\end{array}$ & 3.2 & MS \\
\hline 2 & $\begin{array}{c}\text { [KIT/SNSYN[N3/TUX]]c1F1-\#\#(GLS=2)-29-35-2-3/CM- } \\
\text { L144(BC2)-29-24-1-2-1-3-\#-3-1 }\end{array}$ & 3.2 & MS \\
\hline 3 & $\begin{array}{c}\text { [KIT/SNSYN[N3/TUX]]c1F1-\#\#(GLS=2)-29-35-2-3/CM- } \\
\text { L144(BC2)-29-24-1-3-1-1-\#-2-1 }\end{array}$ & 3.2 & MS \\
\hline 4 & $\begin{array}{c}\text { [KIT/SNSYN[N3/TUX]]c1F1-\#\#(GLS=2)-29-35-2-3/CM- } \\
\text { L144(BC2)-29-24-1-3-2-2-\#-1-1 }\end{array}$ & 2.1 & MR \\
\hline 5 & $\begin{array}{c}\text { [KIT/SNSYN[N3/TUX]]c1F1-\#\#(GLS=2)-29-35-2-3/CM- } \\
\text { L144(BC2)-29-24-1-3-3-1-\#-1-1 }\end{array}$ & 2.4 & MR \\
\hline
\end{tabular}

Among 33 QPM maize lines, 6 (18.2\%) lines showed resistant responses for Common leaf rust at Ambo, in 2015 (Table 2). From 33 QPM maize lines, 18 (54.5\%) lines showed moderately resistant (MR) responses at ambo in the same experimental season. Moderately susceptible (MS) responses were recorded on 6 maize lines. Whereas, three QPM maize lines (SADVLA/CML154 BC2F54-4-1-B-\#-4-\#, P502 SR/CML384X176.....98-2-1-2 BC2F41-3-B-\#-\#-\# and CML176) were showed susceptible (S) responses for common leaf rust at Ambo, in 2015 (Table 2). 


\section{Agricultural Research \& Technology: Open Access Journal}

\begin{tabular}{|c|c|c|c|}
\hline 6 & $\begin{array}{c}\text { [KIT/SNSYN[N3/TUX]]c1F1-\#\#(GLS=2)-29-35-2-3/CM- } \\
\text { L144(BC2)-29-24-1-3-3-3-\#-2-1 }\end{array}$ & 2.2 & $\mathrm{MR}$ \\
\hline 7 & $\begin{array}{l}\text { [POOL9Ac7-SR(BC2)]FS211-1SR-1-1-1-\#/ } \\
\text { CML144(BC2)-14-21-1-3-2-2-\#-1-1 }\end{array}$ & 2.2 & $\mathrm{MR}$ \\
\hline 8 & $\begin{array}{l}\text { [POOL9Ac7-SR(BC2)]FS211-1SR-1-1-1-\#/ } \\
\text { CML144(BC2)-14-21-2-1-4-1-\#-2-1 }\end{array}$ & 2.3 & MR \\
\hline 9 & $\begin{array}{l}\text { [POOL9Ac7-SR(BC2)]FS211-1SR-1-1-1-\#/ } \\
\text { CML144(BC2)-14-21-2-4-5-2-\#-4-1 }\end{array}$ & 2.2 & $\mathrm{MR}$ \\
\hline 10 & $\begin{array}{l}\text { [POOL9Ac7-SR(BC2)]FS211-1SR-1-1-1-\#/ } \\
\text { CML144(BC2)-14-8-4-2-1-3-\#-1-1 }\end{array}$ & 2.5 & $\mathrm{MR}$ \\
\hline 11 & $\begin{array}{l}\text { [POOL9Ac7-SR(BC2)]FS211-1SR-1-1-1-\#/ } \\
\text { CML144(BC2)-14-8-4-2-2-1-\#-2-1 }\end{array}$ & 3.2 & MS \\
\hline 12 & $\begin{array}{l}\text { [POOL9Ac7-SR(BC2)]FS211-1SR-1-1-1-\#/ } \\
\text { CML144(BC2)-14-8-4-2-2-4-\#-3-1 }\end{array}$ & 1.6 & $\mathrm{R}$ \\
\hline 13 & $\begin{array}{l}\text { [POOL9Ac7-SR(BC2)]FS211-1SR-1-1-1-\#/ } \\
\text { CML144(BC2)-14-8-4-2-3-3-\#-1-1 }\end{array}$ & 2.2 & MR \\
\hline 14 & $\begin{array}{l}\text { [POOL9Ac7-SR(BC2)]FS211-1SR-1-1-1-\#/ } \\
\text { CML144(BC2)-14-8-4-2-4-1-\#-1-1 }\end{array}$ & 2.2 & $\mathrm{MR}$ \\
\hline 15 & $\begin{array}{l}\text { [POOL9Ac7-SR(BC2)]FS211-1SR-1-1-1-\#/ } \\
\text { CML144(BC2)-14-8-4-3-2-4-\#-1-1 }\end{array}$ & 2.1 & MR \\
\hline 16 & $\begin{array}{l}\text { [POOL9Ac7-SR(BC2)]FS211-1SR-1-1-1-\#/ } \\
\text { CML144(BC2)-14-8-4-3-3-4-\#-2-1 }\end{array}$ & 2.1 & MR \\
\hline 17 & $\begin{array}{l}\text { [POOL9Ac7-SR(BC2)]FS211-1SR-1-1-1-\#/ } \\
\text { CML144(BC2)-14-8-4-3-4-2-\#-3-1 }\end{array}$ & 1.5 & $\mathrm{R}$ \\
\hline 18 & $\begin{array}{l}\text { [POOL9Ac7-SR(BC2)] FS48-1-1-1-1-1-\#/CM- } \\
\text { L144(BC2)-6-25-2-2-4-1-\#-1-1 }\end{array}$ & 1.8 & $\mathrm{R}$ \\
\hline 19 & $\begin{array}{l}\text { [POOL9Ac7-SR(BC2)] FS48-1-1-1-1-1-\#/CM- } \\
\text { L144(BC2)-6-25-2-2-4-1-\#-5-1 }\end{array}$ & 1.9 & $\mathrm{R}$ \\
\hline 20 & [POOL9Ac7-SR(BC2)] FS48-1-1-3-1-\#/CML144(BC2)-15-8-1-1-3-2-\#-1-1 & 2.2 & MR \\
\hline 21 & [POOL9Ac7-SR(BC2)] FS59-2-2-1-1-\#/CML144(BC2)-9-5-2-1-3-1-\#-4 & 2.4 & MR \\
\hline 22 & $\begin{array}{c}\text { [KIT/SNSYN[N3/TUX]]c1F1-\#\#(GLS=2.5)-32-1-1-\#/CML176BC1F1-12- } \\
\text { 1-3-2-1-\#-1-B }\end{array}$ & 2.3 & MR \\
\hline 23 & $\begin{array}{c}\text { [KIT/SNSYN[N3/TUX]]c1F1-\#\#(GLS=2.5)-32-1-1-\#/CML176BC1F1-12- } \\
1-3-4-2-\#-1-\mathrm{B}\end{array}$ & 1.5 & $\mathrm{R}$ \\
\hline 24 & [POOL9Ac7-SR(BC2)] FS60-2-1-1-1-\#/CML176(BC2)-1-3-2-3-3-\#-2-B & 2.4 & MR \\
\hline 25 & [POOL9Ac7-SR(BC2)] FS60-2-1-1-1-\#/CML176(BC2)-1-3-2-3-4-\#-1-B & 2.3 & MR \\
\hline 26 & [POOL9Ac7-SR(BC2)] FS60-2-1-1-1-\#/CML176(BC2)-1-3-2-3-5-\#-1-B & 1.5 & $\mathrm{R}$ \\
\hline 27 & [POOL9Ac7-SR(BC2)] FS60-2-3-1-3-1-\#/CML176(BC2)-3-1-3-3-3-\#-1-B & 2.3 & MR \\
\hline 28 & SADVLA/CML154 BC2F54-4-1-B-\#-4-\# & 4.5 & S \\
\hline 29 & SADVLA/CML154 BC2F41-1-12-B-\#-\#-\# & 3.2 & MS \\
\hline 30 & SADVLA/CML154 BC2F37-2-1-B-\#-\#-\# & 2.7 & MR \\
\hline 31 & P502 SR/CML384X176.....98-2-1-2 BC2F4-1-3-B-\#-\#-\# & 4 & S \\
\hline 32 & CML176 & 4.5 & $\mathrm{~S}$ \\
\hline 33 & CML491 & 3.5 & MS \\
\hline
\end{tabular}

Table 3: Reaction of Maize (Normal) lines for Common leaf rust disease at Ambo, in 2015.

\begin{tabular}{|c|c|c|c|}
\hline S.no & Maize Lines & Severity (1-5 Scale) & Line Response \\
\hline 1 & B.T.Z.T.V.C -43-B -2-2 -2-\#-1-\#-\#-\#-\# & 1.3 & $\mathrm{R}$ \\
\hline 2 & B.T.Z.T.V.C -43-B -2-2 -3-2-2-1-\#-\#-\# & 1.2 & $\mathrm{R}$ \\
\hline 3 & B.T.Z.T.R.L -83-B-1-3-1-3-1-1-\#-\#-\# & 1.4 & $\mathrm{R}$ \\
\hline 4 & B.T.Z.T.R.L -83-B-1-3-1-3-1-2-\#-\#-\# & 1.5 & $\mathrm{R}$ \\
\hline 5 & B.T.Z.T.R.L -83-B-1-3-1-3-1-3-\#-\#-\# & 3.2 & $\mathrm{MS}$ \\
\hline 6 & B.T.Z.T.V.C -43-B -2-3 -1-1-1-1-\#-\#-\# & 2.2 & $\mathrm{MR}$ \\
\hline
\end{tabular}




\section{Agricultural Research \& Technology: Open Access Journal}

\begin{tabular}{|c|c|c|c|}
\hline 7 & B.T.Z.T.V.C -43-B -2-3 -1-3-2-\#-\#-\#-\# & 1.2 & $\mathrm{R}$ \\
\hline 8 & B.T.Z.T.V.C -43-B -2-1 -1-2-2-\#-\#-\#-\# & 1.4 & $\mathrm{R}$ \\
\hline 9 & B.T.Z.T.V.C -43-B -2-1 -1-3-1-1-\#-\#-\# & 1.2 & $\mathrm{R}$ \\
\hline 10 & B.T.Z.T.V.C -43-B-1-2 -1-1-1-\#-\#-\#-\# & 1.3 & $\mathrm{R}$ \\
\hline 11 & B.T.Z.T.V.C -43-B-1-2 -1-2-1-\#-\#-\#-\# & 1.2 & $\mathrm{R}$ \\
\hline 12 & B.T.Z.T.V.C -99-B-1-2 -1-1-1-\#-\#-\#-\# & 2.7 & MR \\
\hline 13 & B.I.Z.T.V.C -83-B-3-3-3-1-\#-\#-\#-\# & 2.3 & MR \\
\hline 14 & B.I.Z.T.V.C -85-B-2-1-3-1-\#-\#-\#-\# & 1.5 & $\mathrm{R}$ \\
\hline 15 & B.I.Z.T.V.C -8-B-1-3-1-\#-\#-\#-\#-\# & 1.6 & $\mathrm{R}$ \\
\hline 16 & B.T.Z.T.V.C -266-B-1-2 -2-2-\#-\#-\#-\#-\# & 1.4 & $\mathrm{R}$ \\
\hline 17 & B.T.Z.T.V.C -138-B-2-3 -2-1-1-\#-\#-\#-\# & 2.3 & MR \\
\hline 18 & B.T.Z.T.V.C -138-B-2-3 -3-1-2-1-\#-\#-\# & 1.5 & $\mathrm{R}$ \\
\hline 19 & B.I.Z.T.V.C -68-B-3 -2-2-1-\#-\#-\#-\# & 1.4 & $\mathrm{R}$ \\
\hline 20 & B.T.Z.T.V.C -171-1-1-2-2-1-\#-\#-\#-\#-\# & 1.3 & $\mathrm{R}$ \\
\hline 21 & B.T.Z.T.V.C -171-1-1-2-2-2-\#-\#-\#-\#-\# & 1.2 & $\mathrm{R}$ \\
\hline 22 & B.T.Z.T.V.C -171-1-1-2-3-2-\#-\#-\#-\#-\# & 4 & S \\
\hline 23 & B.T.Z.T.V.C -172-1-1-3-1-1-2-\#-\#-\#-\# & 1.3 & $\mathrm{R}$ \\
\hline 24 & B.T.Z.T.V.C -172-1-1-3-1-2-1-\#-\#-\#-\# & 1.5 & $\mathrm{R}$ \\
\hline 25 & B.T.Z.T.V.C -172-1-1-3-1-2-2-\#-\#-\#-\# & 2.2 & MR \\
\hline 26 & B.T.Z.T.V.C -172-1-1-3-2-2-1-1-\#-\#-\# & 3.1 & MS \\
\hline 27 & B.T.Z.T.V.C -172-1-1-3-2-2-1-2-\#-\#-\# & 2.1 & MR \\
\hline 28 & B.T.Z.T.V.C -172-1-1-3-3-1-1-\#-\#-\#-\# & 1.2 & $\mathrm{R}$ \\
\hline 29 & SINT T.SR.B.T.Z.T.4P-1P-4P-1P-3P-6-1-1-1-\#-\#-\# & 1.3 & $\mathrm{R}$ \\
\hline 30 & SINT TSR.B.T.Z.T.19P-1P-1P-2P-1P-5-1-1-3-\#-\#-\# & 2.6 & MR \\
\hline 31 & B-62.5\%9A TSR-19P-3P-1P-2P-1P-1P-2-1-\#-2-\#-\#-\# & 1.4 & $\mathrm{R}$ \\
\hline 32 & B.T.Z.T.V.C.PR.93A 1-2P-1-2-1 -4-2-1-\#-\#-\#-\# & 1.7 & $\mathrm{R}$ \\
\hline
\end{tabular}

Among 32 normal maize lines, 22 (68.8\%) lines showed resistant responses for Common leaf rust at Ambo, in 2015 (Table 3). Moderately resistant responses were recorded on seven normal maize lines. Out of 32 normal maize lines, moderately susceptible responses were recorded on B.T.Z.T.V.C -172-1-1-3-2-2-1-1-\#\#-\# and B.T.Z.T.R.L -83-B-1-3-1-3-1-3-\#-\#-\# normal maize lines. Susceptible response was recorded onB.T.Z.T.V.C -171-1-1-2-3-2\#-\#-\#-\#-\# maize line at Ambo, in 2015.

Table 4: Responses of maize lines for TLB disease at Ambo, in 2016.

\begin{tabular}{|c|c|c|c|c|c|c|c|}
\hline $\begin{array}{c}\text { QPM maize } \\
\text { Line }\end{array}$ & $\begin{array}{c}\text { Severity (1-5 } \\
\text { Scale) }\end{array}$ & AUDPC & Line Responses & Normal Maize Line & $\begin{array}{c}\text { Severity (1-5 } \\
\text { Scale) }\end{array}$ & AUDPC & Line Responses \\
\hline $\begin{array}{c}\text { AM- } \\
\text { B15QTWP3-2 }\end{array}$ & $\mathbf{2 . 0 3 c}$ & $\mathbf{5 8 . 7 2 b c d e}$ & MR & AMB15N-37LD-17 & $\mathbf{2 . 1 8 b}$ & $\mathbf{6 4 . 8 4 e f g}$ & MR \\
\hline AMB15QTWP3-4 & $2.05 \mathrm{c}$ & $67.79 \mathrm{bcd}$ & MR & AMB15N-37LD-24 & $1.04 \mathrm{c}$ & $7.69 \mathrm{~h}$ & R \\
\hline AMB15QTWP3-5 & $1.32 \mathrm{~d}$ & $17.09 \mathrm{f}$ & R & AMB15N-37LD-26 & $3.25 \mathrm{a}$ & $136.11 \mathrm{~b}$ & MS \\
\hline AMB15QTWP3-8 & $1.08 \mathrm{~d}$ & $14.30 \mathrm{f}$ & R & AMB15N-37LD-27 & $3.6 \mathrm{a}$ & $171.18 \mathrm{ab}$ & S \\
\hline $\begin{array}{c}\text { AM- } \\
\text { B15QTWP3-11 }\end{array}$ & $2.04 \mathrm{c}$ & $53.98 \mathrm{bcde}$ & MR & AMB15N-37LD-35 & $1.22 \mathrm{c}$ & $9.14 \mathrm{gh}$ & R \\
\hline $\begin{array}{c}\text { AM- } \\
\text { B15QTWP3-14 }\end{array}$ & $2.09 \mathrm{bc}$ & $65.07 \mathrm{bcd}$ & MR & AMB15N-37LD-36 & $3.25 \mathrm{a}$ & $135.06 \mathrm{~b}$ & MS \\
\hline $\begin{array}{c}\text { AM- } \\
\text { B15QTWP3-15 }\end{array}$ & $1.28 \mathrm{~d}$ & $15.44 \mathrm{f}$ & R & AMB15N-37LD-48 & $3.12 \mathrm{a}$ & $128.08 \mathrm{bcd}$ & MS \\
\hline
\end{tabular}


Agricultural Research \& Technology: Open Access Journal

\begin{tabular}{|c|c|c|c|c|c|c|c|}
\hline $\begin{array}{c}\text { AM- } \\
\text { B15QTWP3-20 }\end{array}$ & $2.73 \mathrm{ab}$ & $112.49 a$ & MR & AMB15N-37LD-49 & $1.25 \mathrm{c}$ & 15.59fgh & $\mathrm{R}$ \\
\hline $\begin{array}{c}\text { AM- } \\
\text { B15QTWP3-21 }\end{array}$ & $2.04 \mathrm{c}$ & $60.58 \mathrm{bcd}$ & MR & AMB15N-37LD-53 & $1.0 \mathrm{c}$ & $7.5 \mathrm{~h}$ & $\mathrm{R}$ \\
\hline $\begin{array}{c}\text { AM- } \\
\text { B15QTWP3-22 }\end{array}$ & $3.05 a$ & $117.34 \mathrm{a}$ & MS & AMB15N-23-39 & $2.17 \mathrm{~b}$ & 73.28de & MR \\
\hline $\begin{array}{c}\text { AM- } \\
\text { B15QTWP3-26 }\end{array}$ & $2.04 \mathrm{c}$ & 41.61cdef & MR & AMB15N-21-1 & $1.01 \mathrm{c}$ & $7.56 \mathrm{~h}$ & $\mathrm{R}$ \\
\hline $\begin{array}{c}\text { AM- } \\
\text { B15QTWP3-28 }\end{array}$ & $2.18 b c$ & 67.19bcd & MR & AMB15N-21-5 & $3.77 \mathrm{a}$ & $196.48 a$ & $\mathbf{S}$ \\
\hline $\begin{array}{c}\text { AM- } \\
\text { B15QTWP3-31 }\end{array}$ & $1.35 \mathrm{~d}$ & $22.38 \mathrm{ef}$ & $\mathrm{R}$ & AMB15N-21-33 & $1.0 \mathrm{c}$ & $7.50 \mathrm{~h}$ & $\mathrm{R}$ \\
\hline $\begin{array}{c}\text { AM- } \\
\text { B15QTWP3-32 }\end{array}$ & $1.26 \mathrm{~d}$ & $16.91 \mathrm{f}$ & $\mathrm{R}$ & AMB15N-21-34 & $2.17 \mathrm{~b}$ & $70.03 \mathrm{ef}$ & MR \\
\hline $\begin{array}{c}\text { AM- } \\
\text { B15QTWP3-33 }\end{array}$ & $2.1 \mathrm{bc}$ & $64.89 \mathrm{bcd}$ & MR & AMB15N-21-42 & $3.16 \mathrm{a}$ & $131.44 \mathrm{bc}$ & MS \\
\hline $\begin{array}{c}\text { AM- } \\
\text { B15QTWP3-36 }\end{array}$ & $1.37 \mathrm{~d}$ & $22.39 \mathrm{ef}$ & $\mathrm{R}$ & AMB15KN20-8 & $2.26 \mathrm{~b}$ & 75.30cde & MR \\
\hline $\begin{array}{c}\text { AM- } \\
\text { B15QTWP3-37 }\end{array}$ & $2.09 \mathrm{bc}$ & $72.67 \mathrm{bc}$ & MR & AMB15EN18-1 & $3.15 \mathrm{a}$ & $129.22 \mathrm{bcd}$ & MS \\
\hline $\begin{array}{c}\text { AM- } \\
\text { B15QTWP3-43 }\end{array}$ & $2.43 a b c$ & $85.05 \mathrm{ab}$ & MR & AMB15EN18-13 & $1.13 \mathrm{c}$ & $9.45 \mathrm{gh}$ & $\mathrm{R}$ \\
\hline $\begin{array}{c}\text { AM- } \\
\text { B15QTWP3-47 }\end{array}$ & $3.05 a$ & $121.09 a$ & MS & AMB15EN18-15 & $1.18 \mathrm{c}$ & 19.59efgh & $\mathrm{R}$ \\
\hline $\begin{array}{c}\text { AM- } \\
\text { B15QTWP3-48 }\end{array}$ & $1.36 \mathrm{~d}$ & 31.17 & $\mathrm{R}$ & AMB15EN18-22 & $1.22 \mathrm{c}$ & $10.58 \mathrm{gh}$ & $\mathrm{R}$ \\
\hline Mean & 1.95 & 56.41 & & & 2.11 & 70.28 & \\
\hline CV (\%) & 15.86 & 31.22 & & & 16.67 & 38.4 & \\
\hline LSD (0.05) & 0.65 & 36.86 & & & 0.74 & 56.49 & \\
\hline
\end{tabular}

Among 20 normal maize lines, 9 lines showed resistant responses for Turcicum leaf blight (TLB) at Ambo, in 2016 (Table 4). Moderately resistant responses were recorded on four normal maize lines. Moderately susceptible responses were recorded on five normal maize lines, and only 2 normal maize lines (AMB15N37LD-27 and AMB15N-21-5) showed susceptible responses for TLB at Ambo, in 2016 (Table 4).

Among 20 QPM maize lines, five lines were showed resistant (R) responses for Common leaf rust (CLR) at Ambo, in 2016 (Table 5). Moderately resistant (MR) and moderately susceptible

Table 5: Responses of maize lines for CLR disease at Ambo, in 2016.
(MS) responses were recorded on ten and four QPM maize lines, respectively. Only one QPM maize line (AMB15QTWP3-32) showed susceptible (S) response for CLR disease at Ambo, in 2016 (Table 5).

Out of 20 normal maize lines, eleven lines showed resistant responses for CLR disease at Ambo, in 2016 (Table 5). Moderately resistant responses were recorded on nine normal maize lines. Relatively higher AUDPC and severity of CLR was recorded on QPM maize lines than normal maize lines at Ambo, in 2016 (Table 5).

\begin{tabular}{|c|c|c|c|c|c|c|c|}
\hline $\begin{array}{c}\text { QPM maize } \\
\text { Line }\end{array}$ & $\begin{array}{c}\text { Severity (1-5 } \\
\text { Scale) }\end{array}$ & AUDPC & Line Responses & $\begin{array}{c}\text { Normal Maize } \\
\text { Line }\end{array}$ & Severity (1-5 scale) & AUDPC & Line Responses \\
\hline $\begin{array}{c}\text { AM- } \\
\text { B15QTWP3-2 }\end{array}$ & $\mathbf{3 . 2 a b}$ & $\mathbf{1 2 9 . 7 a b c d}$ & MS & $\begin{array}{c}\text { AMB15N- } \\
\mathbf{3 7 L D - 1 7}\end{array}$ & $\mathbf{1 . 0 1 d}$ & $\mathbf{7 . 5 3 d}$ & $\mathbf{R}$ \\
\hline AMB15QTWP3-4 & $1.5 \mathrm{f}$ & $32.9 \mathrm{gh}$ & $\mathrm{R}$ & $\begin{array}{c}\text { AMB15N- } \\
37 \mathrm{LD}-24\end{array}$ & $2.1 \mathrm{c}$ & $76.36 \mathrm{bc}$ & $\mathrm{MR}$ \\
\hline AMB15QTWP3-5 & $1.2 \mathrm{fg}$ & $9.6 \mathrm{~h}$ & $\mathrm{R}$ & $\begin{array}{c}\text { AMB15N- } \\
37 \mathrm{LD}-26\end{array}$ & $1.0 \mathrm{~d}$ & $7.5 \mathrm{~d}$ & $\mathrm{R}$ \\
\hline AMB15QTWP3-8 & $3.4 \mathrm{a}$ & $162.7 \mathrm{ab}$ & MS & $\begin{array}{c}\text { AMB15N- } \\
37 \mathrm{LD}-27\end{array}$ & 1.0d & $7.5 \mathrm{~d}$ & $\mathrm{R}$ \\
\hline $\begin{array}{c}\text { AM- } \\
\text { B15QTWP3-11 }\end{array}$ & $2.7 \mathrm{~cd}$ & $104.7 \mathrm{cde}$ & MR & $\begin{array}{c}\text { AMB15N- } \\
37 \mathrm{LD}-35\end{array}$ & $2.31 \mathrm{bc}$ & $87.58 \mathrm{abc}$ & MR \\
\hline $\begin{array}{c}\text { AM- } \\
\text { B15QTWP3-14 }\end{array}$ & $2.2 \mathrm{e}$ & $73.1 \mathrm{efg}$ & MR & $\begin{array}{c}\text { AMB15N- } \\
37 \mathrm{LD}-36\end{array}$ & $2.5 \mathrm{ab}$ & $92.79 \mathrm{ab}$ & MR \\
\hline
\end{tabular}




\section{Agricultural Research \& Technology: Open Access Journal}

\begin{tabular}{|c|c|c|c|c|c|c|c|}
\hline $\begin{array}{c}\text { AM- } \\
\text { B15QTWP3-15 }\end{array}$ & $2.7 \mathrm{~cd}$ & $103.3 \mathrm{cde}$ & MR & $\begin{array}{l}\text { AMB15N- } \\
37 \mathrm{LD}-48\end{array}$ & $2.38 \mathrm{abc}$ & $80.08 \mathrm{abc}$ & MR \\
\hline $\begin{array}{c}\text { AM- } \\
\text { B15QTWP3-20 }\end{array}$ & $3.3 \mathrm{a}$ & $148.7 \mathrm{abc}$ & MS & $\begin{array}{l}\text { AMB15N- } \\
37 \mathrm{LD}-49\end{array}$ & $1.03 \mathrm{~d}$ & $7.75 \mathrm{~d}$ & $\mathrm{R}$ \\
\hline $\begin{array}{c}\text { AM- } \\
\text { B15QTWP3-21 }\end{array}$ & $2.6 \mathrm{~cd}$ & 99.8de & MR & $\begin{array}{l}\text { AMB15N- } \\
37 \mathrm{LD}-53\end{array}$ & $1.05 \mathrm{~d}$ & $7.81 \mathrm{~d}$ & $\mathrm{R}$ \\
\hline $\begin{array}{c}\text { AM- } \\
\text { B15QTWP3-22 }\end{array}$ & 2.4cde & 83.9def & MR & AMB15N-23-39 & $2.18 \mathrm{bc}$ & $66.60 c$ & MR \\
\hline $\begin{array}{c}\text { AM- } \\
\text { B15QTWP3-26 }\end{array}$ & $1 \mathrm{~g}$ & $41.3 \mathrm{fgh}$ & $\mathrm{R}$ & AMB15N-21-1 & $1.0 \mathrm{~d}$ & $7.5 \mathrm{~d}$ & $\mathrm{R}$ \\
\hline $\begin{array}{c}\text { AM- } \\
\text { B15QTWP3-28 }\end{array}$ & $2.8 \mathrm{bc}$ & 118.5bcde & MR & AMB15N-21-5 & $1.0 \mathrm{~d}$ & $7.5 \mathrm{~d}$ & $\mathbf{R}$ \\
\hline $\begin{array}{c}\text { AM- } \\
\text { B15QTWP3-31 }\end{array}$ & $1 \mathrm{~g}$ & $7.5 \mathrm{~h}$ & $\mathrm{R}$ & AMB15N-21-33 & $1.0 \mathrm{~d}$ & $7.5 \mathrm{~d}$ & $\mathrm{R}$ \\
\hline $\begin{array}{c}\text { AM- } \\
\text { B15QTWP3-32 }\end{array}$ & $1 \mathrm{~g}$ & $7.5 \mathrm{~h}$ & $\mathrm{R}$ & AMB15N-21-34 & $1.0 \mathrm{~d}$ & $7.5 \mathrm{~d}$ & $\mathrm{R}$ \\
\hline $\begin{array}{c}\text { AM- } \\
\text { B15QTWP3-33 }\end{array}$ & $3.6 \mathrm{a}$ & $171.03 a$ & S & AMB15N-21-42 & $2.7 \mathrm{a}$ & $107.72 \mathrm{a}$ & MR \\
\hline $\begin{array}{c}\text { AM- } \\
\text { B15QTWP3-36 }\end{array}$ & $2.1 \mathrm{e}$ & 78.7efg & MR & AMB15KN20-8 & $2.16 \mathrm{bc}$ & $64.58 \mathrm{c}$ & MR \\
\hline $\begin{array}{c}\text { AM- } \\
\text { B15QTWP3-37 }\end{array}$ & $3.4 \mathrm{a}$ & $154.4 \mathrm{ab}$ & MS & AMB15EN18-1 & $2.18 \mathrm{bc}$ & $79.73 \mathrm{bc}$ & MR \\
\hline $\begin{array}{c}\text { AM- } \\
\text { B15QTWP3-43 }\end{array}$ & $2.4 \mathrm{de}$ & 84.6def & MR & $\begin{array}{c}\text { AM- } \\
\text { B15EN18-13 }\end{array}$ & $2.12 \mathrm{bc}$ & $69.27 \mathrm{bc}$ & MR \\
\hline $\begin{array}{c}\text { AM- } \\
\text { B15QTWP3-47 }\end{array}$ & $2.1 \mathrm{e}$ & 78efg & MR & $\begin{array}{c}\text { AM- } \\
\text { B15EN18-15 }\end{array}$ & $1.03 \mathrm{~d}$ & $7.73 d$ & $\mathrm{R}$ \\
\hline $\begin{array}{c}\text { AM- } \\
\text { B15QTWP3-48 }\end{array}$ & $3.5 \mathrm{a}$ & $177.4 \mathrm{a}$ & MR & $\begin{array}{c}\text { AM- } \\
\text { B15EN18-22 }\end{array}$ & $1.0 \mathrm{~d}$ & $7.5 \mathrm{~d}$ & $\mathrm{R}$ \\
\hline Mean & 2.4 & 93.4 & & & 1.59 & 40.8 & \\
\hline CV (\%) & 8.1 & 24.9 & & & 11.49 & 29.95 & \\
\hline LSD (0.05) & 0.41 & 48.6 & & & 0.38 & 25.57 & \\
\hline
\end{tabular}

\section{Conclusion and Recommendation}

Turcicum leaf blight (TLB) and Common leaf rust (CLR) is among the major foliar diseases of maize in Ethiopia. Screening was done at Ambo (TLB and CLR) and Bako (TLB only) for two consecutive years (2015 and 2016 growing seasons) in order to know the responses of maize lines (QPM and Normal maize lines) for two diseases. This was done on 73 maize lines (53 QPM and 20 Normal) for TLB, and on 105 maize lines (53 QPM and 52 normal) for CLR.

Among 33 tested QPM maize lines, 12 (36.3\%) lines were showed resistant responses for Turcicum leaf blight in 2015, only at Ambo. From 33 maize lines, 14 (42.4\%) lines showed resistant responses both at Ambo and Bako, in the same growing season. Moderately, responses were recorded at Ambo and Bako on seven and eighteen maize lines in 2015, respectively. Only one QPM maize line showed susceptible response at Bako experimental site. Out of 40 maize lines (20 QPM, and 20 Non-QPM), resistant responses were recorded on 7 QPM and 9 normal maize lines for TLB disease in 2016, at Ambo. Two non-QPM maize lines were showed susceptible responses for TLB disease at Ambo, in the same growing season, 2016.

Among 33QPM maize lines, 6 (18.2\%) line showed resistant responses for CLR disease in 2015 at Ambo. Susceptible responses were recorded on 3 QPM maize lines for CLR disease at Ambo in the same growing season. Out of 32 normal maize lines, 22 (68.7\%) lines showed resistant responses; and susceptible response was recorded on 1 line in 2015, at Ambo. Among 40 maize lines (20 $\mathrm{QPM}$, and 20 Non-QPM), resistant responses were recorded on 5 QPM and 11 normal maize lines in 2016, at Ambo. One QPM-maize line was showed susceptible response for CLR disease at Ambo, in the same growing season.

In general, higher mean severity and mean AUDPC of CLR was recorded on QPM than non-QPM (normal) maize lines whereas higher mean severity and mean AUDPC of TLB was recorded on non-QPM than QPM maize lines. Therefore, attention should be given for both diseases during screening in order to develop resistant maize varieties for both maize types. Those selected resistance maize lines from this screening should be used in breeding program and finding of resistant maize lines for both diseases will be continued using conventional and molecular methods.

\section{Acknowledgement}

I would like to thank Ethiopian Institute of Agricultural research and Ambo Plant Protection Research Center for providing support in different aspects during the research time. 


\section{References}

1. Randjelovic V, Prodanovic S, Tomic Z, Simic A (2011) Genotype X year effect on grain yield and nutritive values of maize (Zea mays L.). J Animal Vet Sci Adv 10(7): 835-840.

2. Bello OB, Abdulmalig SY, Afolabi MS, Lge SA (2010) Correlation and path coefficient hybrids analysis of yield and agronomic characters among open pollinated maize varieties and their F1in a diallel cross. Afr J Biotech 9(18): 2633-2639.

3. CSA (Central Sastical Authority) (2015) Agriculture sample survey report on area and production for major crops for 2014/015. The FDRE Sastical Buletin. Addis Ababa, Ethiopia.

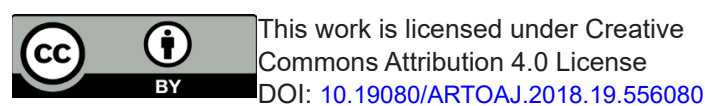

4. Renfro BL, Ullstrup AJ (1996) Comparison of maize disease in temperate and tropical environments. PANS 22(4): 491-498.

5. Durable Rust Resistance in Wheat (DRRW) Phase II (2010) Proposal for Bill and Melinda Gates Foundation. Cornell University Office of International Programs, USA, pp. 413.

6. Payak MM, Sharma RC (1982) Maize diseases and approaches to their management in India. Trop Pest Management 31(4): 302-310.

7. Wilcoxson RD, Skovmand B, Atif AH (1975) Evaluation of wheat cultivars ability to retard development of stem rust. Annals of Applied Biology 80(3): 275-2181.

8. Gomez KA, Gomez AA (1984) Statistical procedures for agricultural research ( $2^{\text {nd }}$ edn), John wiley and sons, New York, USA, pp. 690.

\section{Your next submission with Juniper Publishers will reach you the below assets}

- Quality Editorial service

- Swift Peer Review

- Reprints availability

- E-prints Service

- Manuscript Podcast for convenient understanding

- Global attainment for your research

- Manuscript accessibility in different formats

( Pdf, E-pub, Full Text, Audio)

- Unceasing customer service

Track the below URL for one-step submission https://juniperpublishers.com/online-submission.php 